\title{
Nobel rewards two laboratory revolutions
}

London. This year's Nobel Prize for Chemistry has been awarded to Michael Smith for his work on site-directed mutagenesis, and Kary B. Mullis for the invention of the polymerase chain reaction (PCR). Both techniques have already revolutionized the procedures used in research laboratories and biopharmaceutical companies.

Site-directed mutagenesis uses mismatched synthetic DNA fragments (oligonucleotides) to alter specific nucleotides along a sequence of DNA. This produces predictable changes in the aminoacid sequence of the translated protein, and makes it possible both to work out the how the different parts of a protein contribute to its function, and eventually to design proteins. PCR also uses oligonucleotides, but in a cycle that lets an extremely small amount of DNA be amplified exponentially. Sitedirected mutagenesis and PCR are often combined.

"We were in a unique position to develop site-directed mutagenesis", says Smith, who works at the University of British Columbia in Canada, referring to work carried out with his colleagues. "We had information on duplex stability with mismatches, and at the time one had to be a chemist to make oligonucleotides."

"Few genomic sequences were known, but I was on sabbatical in Fred Sanger's lab, and so had the sequence of the bacteriophage $\Phi \mathrm{X}-174$, which was relatively easy to work with because it was single stranded DNA." The prize is "a great honour" he says, be-

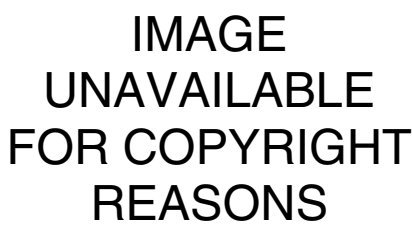

Michael Smith: "in a unique position to develop site-directed mutagenesis".

cause "there are so many scientists doing good things".

Kary Mullis, who is now a freelance consultant based in La Jolla, California, was stirred but not shaken by news of his prize: "From what people told me, I figured it would happen eventually, and it might as well be sooner rather than later."

Mullis says that the prize recognizes the significance of a finding and not the work of individuals during their lifetime. "Whether I'm a maverick, weirdo, or whatever, if the finding is important, then it should get the Nobel prize."

Alan Fersht, a 'protein engineer' who is professor of organic chemistry at the University of Cambridge, says he is delighted that gene technology had been honoured by the chemistry prize. "This is modern organic chemistry; for both techniques you need chemistry to make the oligonucleotides and enzymes to finish it off." He says that "sitedirected mutagenesis has totally transformed my science".

\section{IMAGE UNAVAILABLE FOR COPYRIGHT REASONS}

\section{Kary B. Mullis: "if the finding's important,} it should get the prize".

Peter Little, a molecular geneticist at Imperial College, London, describes Smith and Mullis as a "surprising pairing of winners", but adds that "there was no doubt that they should win the Nobel prize". He welcomes the award for PCR because "it has changed the way molecular biology is done", and made the public more aware of the possibilities of DNA analysis. He says that site-directed mutagenesis is "an incredibly important tool" and that "we have not yet had time to see its full effects on medicine".

Alec Jeffreys, who invented DNA-fingerprinting in his laboratory at the University of Leicester, has used PCR for many applications - including its first use to identify a murder victim. He says he now finds it impossible to conceive of a molecular genetics laboratory without PCR. "Like all good scientific ideas, it's exquisitely simple and, in retrospect, obvious", he says. "I'm sure that thousands of people like myself are kicking themselves that they didn't think of it." Kimberley Carr

\section{Prize lifts prospects for gravity wave detectors}

London. The Nobel Prize for Physics has been awarded to Joseph Taylor and Russell Hulse of Princeton University for their discovery of binary pulsars. This is the second time that the physics prize has gone to research on pulsars - rotating neutron stars that send a lighthouse-like beam of radiation sweeping through space.

Pulsars were discovered in 1967 by Anthony Hewish of the University of Cambridge and his student Jocelyn Bell. Their observation of oscillations from pulsars at radio frequencies confirmed that when a star explodes in a supernova, electrons can fuse with protons in a highly dense, imploding core to create a compact body composed only of neutrons. Some neutron stars were predicted to have a strong magnetic field, which would cause them to emit intense beams of radiation at each magnetic pole. If the star rotates, the beam appears to turn on and off when viewed from Earth.

While the implications of this discovery lay primarily with stellar and nuclear physics, Taylor and Hulse's observation of a pulsar (PSR1913+16) circulating a companion in a binary system confirmed an important prediction of Einstein's theory of general relativity: that a rotating mass should emit gravitational energy as waves in much the same way as a rotating electric charge emits electromagnetic radiation. This had never been shown experimentally, because the amount of energy emitted is so small.

In 1919, Arthur Eddington provided confirmation of Einstein's theory that a gravitational field represents a curvature of spacetime. Taking the opportunity offered by an eclipse, he observed that light rays from a star are "bent" by the Sun's gravitational field, as the theory predicted.

Taylor and Hulse reasoned that they should be able to detect gravitational waves from their binary pulsar because of the close orbits of the pair - as the binary loses orbital energy, the pulsar's orbit spirals inwards and the period decreases. By 1978 , Taylor's group had confirmed that the orbit of PSR 1913+16 decreased by the predicted amount of 75 microseconds per year (within errors of 20 per cent).

It was this quantitative agreement (now with much tighter error margins) that provided the compelling test of the theory, says Clifford Will of Washington University, in St Louis, Missouri, who is a specialist on tests of general relativity. He says that the discovery of a binary pulsar was somewhat fortuitous, in that it would have been undetectable had the signal been slightly weaker. But he praises Hulse's persistence in pursuing his observations instead of rejecting them as instrumental artefacts.

Hulse says he was more disappointed than excited when he first noticed that the pulsar's period appeared to vary between observations. He says that with further observations, he became convinced that this variation was not an instrumental artefact, and that he eventually realized it was due to the presence of a companion body.

Although Taylor stresses that he and Hulse had no intention of "setting out to work on relativity", he explains that the 